\title{
La resolución de problemas aritméticos en el aula. ¿Qué hacen los profesores cuando trabajan conjuntamente con sus alumnos?
}

\author{
Javier Rosales, Josetxu Orrantia, Santiago Vicente

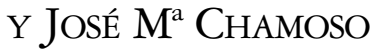 \\ Universidad de Salamanca
}

\begin{abstract}
Resumen
En este artículo se analiza la interacción que tres profesores en servicio y tres profesores en formación mantienen con sus alumnos cuando resuelven problemas de matemáticas. Para ello se parte, por un lado, de los modelos teóricos que describen los procesos cognitivos implicados en la resolución de problemas, y por otro, de un sistema de análisis que nos permite conocer en quéparte del proceso de resolución se centra en cada momento la interacción y en qué medida es el profesor, el alumno o ambos, quienes toman la responsabilidad en la construcción de los contenidos que se hacen públicos en la interacción. Los resultados muestran que los profesores en servicio resuelven los problemas únicamente tras asegurar la comprensión del problema, y permiten una participación mayor de los alumnos en los aspectos centrales de la comprensión del problema, mientras que los profesores en formación no aseguran la comprensión del problema antes de elegir la operación a realizar y son más directivos en los aspectos centrales de la comprensión.
\end{abstract}

Palabras clave: Análisis de la interacción, profesores en servicio y en formación, resolución de problemas

\section{Arithmetic problem solving in the classroom: What do teachers do when they work jointly with students?}

\begin{abstract}
The interaction between three in-service teachers and three pre-service teachers with their pupils when solving compare word problems is analysed. In order to do so, the theoretical frame is based, on the one band, on the theoretical models that describe the cognitive processes involved in word problem solving, and on the other, on an interaction analysis system that allows us to know the part of the word problem solving process on which each moment of the interaction is focused, and also who is taking the main responsibility for creating the contents made public in the interaction, namely, the teacher, the pupil or both. The results showed that inservice teachers solved word problems only when they were sure that the pupils bad understood the problem, and also they allowed pupils greater participation in central activities involving comprehension. However, preservice teachers did not make sure that pupils' had understood the problem before choosing the operation needed to solve it, and were more directive in central aspects involving compression.
\end{abstract}

Keywords: Classroom interaction analysis, in-service and pre-service teachers, word problem solving.

Agradecimentos: Esta investigación ha sido financiada por los proyectos SA127A06, concedido por la Junta de Castilla y León, y por el proyecto SEJ2006/11249, del Ministerio de Educación y Ciencia.

Correspondencia con los autores: Javier Rosales. Universidad de Salamanca. Facultad de Educación. Pso. Canalejas 169.37008 Salamanca.E-mail: rosales@usal.es

Original recibido: Julio, 2008. Aceptado: Septiembre, 2008. 
Es indudable que cada vez resulta más importante para la investigación educativa la descripción de los contextos socioculturales en los que se desarrollan los aprendizajes. En este sentido, a nadie extraña que, cada vez con más frecuencia, vayan apareciendo trabajos que hacen suya esta preocupación. Muestra de este interés es este mismo Monográfico sobre Resolución de Problemas en el que nuestra contribución consiste en presentar algunos de los resultados más sobresalientes que hemos encontrado cuando hemos estudiado la interacción entre profesor y alumnos. En concreto, nuestra aportación pretende clarificar cuál es el papel que pueden jugar las teorías cognitivas sobre la resolución de problemas en el estudio de lo que ocurre en las aulas. Para ello, hemos dedicado una primera parte del trabajo a justificar desde dónde hemos abordado el estudio de la interacción y, en una segunda, presentaremos algunos de los resultados que mejor clarifican nuestra decisión de tomar en consideración las aportaciones de la psicología cognitiva al estudio de la práctica educativa.

El estudio de las prácticas educativas desarrolladas en las clases de matemáticas ha prestado una atención especial a las ayudas que los profesores ponen en marcha durante la resolución de las tareas matemáticas así como al papel que desempeñan profesores y alumnos en su realización. Por ejemplo, Williams y Baxter (1996) diferencian entre dos tipos de ayudas instruccionales: las que van dirigidas a la resolución de las tareas (analytic scaffolding) y las que van dirigidas al desarrollo de normas sociales de comportamiento y uso del discurso en el aula (social scaffolding). Por su parte, Hatano y Inagaki (1991), al hablar del papel que juegan profesores y alumnos en la realización de las tareas, diferencian entre aquellos casos en los que la información en las aulas fluye de forma vertical: cuando se transmite desde las personas más competentes hacia las menos competentes o de forma horizontal: cuando la información se transmite entre personas del mismo nivel de competencia.

En nuestro caso, estamos interesados en describir cómo son las interacciones que mantienen profesores y alumnos cuando tratan de resolver conjuntamente problemas aritméticos. Específicamente, nuestro interés se ha centrado más bien en lo que Williams y Baxter han denominado analytic scaffolding (el trabajo de Nuria Planas en este mismo Monográfico es un buen ejemplo de lo que sería un social scaffolding). Del mismo modo, también estamos interesados en el papel que juegan, tanto los profesores como sus alumnos en la resolución de problemas aritméticos. En este sentido, nos interesa describir cuál es la autonomía o responsabilidad que asumen los profesores y sus alumnos en la realización de esta tarea. O lo que es lo mismo, estamos más interesados por lo que Hatano y Inagaki (1991) han denominado interacciones verticales entre profesor y alumnos y menos por las interacciones de tipo horizontal que se producen entre grupos de alumnos.

Para estudiar qué se hace en las aulas hemos comparado grupos de competencia bien delimitados; profesores en ejercicio y futuros profesores. Las diferencias entre ambos grupos nos han permitido profundizar en la comprensión de los procesos cognitivos inherentes a la profesión docente tan necesarios para la promoción y mejora de las prácticas profesionales de los profesores. Por ejemplo, en este sentido, en una revisión reciente, Hogan, Rabinowitz y Craven (2003) han recopilado algunas de las diferencias más importantes entre el comportamiento de profesores expertos y principiantes en contextos de enseñanza-aprendizaje. De forma más concreta, entre otras diferencias, han señalado que los profesores expertos ajustan de una forma más precisa sus ayudas a las características específicas de sus alumnos, mientras que los profesores principiantes tienden a ajustar sus ayudas a la clase de una forma mucho más inespecífica. En definitiva, el estudio de la práctica educativa en general y del comportamiento de profesores 
expertos y principiantes nos puede ayudar a describir el modo en que ambos grupos de competencia representan sus propias prácticas profesionales.

\section{EL ESTUDIO DEL DISCURSO EN LAS AULAS DE MATEMÁTICAS}

La investigación educativa ha repetido insistentemente que una buena parte de los conocimientos que aprenden los alumnos en las aulas son desarrollados en contextos comunicativos de interacción. Por este motivo, el estudio del discurso de profesores y alumnos ha sido uno de los elementos más utilizados para describir cómo se producen, realmente, los aprendizajes en las aulas (Mercer y Littleton, 2007). Cómo es lógico, el caso de las matemáticas no ha sido una excepción.

Sin embargo, a pesar de la coincidencia en el punto de partida, y como es fácil de imaginar, la forma en que se ha abordado el estudio de la vida en las aulas en general y de la interacción profesor-alumnos en particular no ha sido monolítica (véase para una revisión sobre esta cuestión el trabajo de Sánchez y Rosales, 2005). Más allá de la consideración de todas las diferencias que pudieran establecerse entre unas y otras formas de actuar, en este momento nos gustaría llamar la atención, específicamente, sobre una de las diferencias más relevantes: la consideración de teorías específicas de dominio frente a teorías genéricas para el análisis e interpretación de la vida en las aulas.

Normalmente, la investigación educativa ha utilizado modelos teóricos genéricos para describir cómo se producen los aprendizajes matemáticos en las aulas. Por ejemplo, en muchos trabajos de corte sociocultural se ha tomado como punto de partida para el análisis de sus resultados lo que se considera un buen aprendizaje y cómo este debería ser promovido ${ }^{1}$. Un ejemplo de este tipo de estudios es el realizado por Nathan y Knuth (2003), quienes analizaron las interacciones que mantuvieron un grupo de alumnos con sus profesores en una clase dedicada al número racional. En el trabajo se estudiaron cuáles fueron los contenidos que se intercambiaron profesores y alumnos y el papel que asumió cada uno de ellos en la elaboración de esos contenidos matemáticos. Para ello, utilizaron un sistema de análisis ad hoc en el que no se consideraba, específicamente, la naturaleza de los contenidos desarrollados en la interacción. Esto es, su análisis partió de una idea genérica sobre lo que supone aprender, pero en ningún caso se tuvo en cuenta lo que supone el aprendizaje del número racional como un contenido matemático específico y con unas características propias.

En nuestro trabajo hemos optado por el uso de modelos teóricos específicos en los que se describen cuáles son los procesos cognitivos implicados en resolución de un problema aritmético (Briars y Larkin, 1984; Kintsch, 1988, 1998; Kintsch y Greeno, 1985; Riley, Greeno y Heller, 1983). En concreto, para analizar la interacción entre profesor y alumnos hemos partido de los modelos que nos explican las operaciones cognitivas que llevamos acabo cuando resolvemos problemas de comparación, de acuerdo con la categorización empleada por Riley et al. (1983) (se puede ver también Fuson, 1992; Kintsch, 1988, 1998; Reed, 1999 o Verschaffel y De Corte, 1997).

Un ejemplo de problema de comparación, tomado de Vicente, Orrantia y Verschaffel (2008) sería el siguiente: "Juan tiene 8 canicas. Pedro tiene 3 canicas menos que Juan. ¿Cuántas canicas tiene Pedro?”. En los problemas de esta categoría se establece una comparación entre dos conjuntos (en el ejemplo, las canicas de Juan y las de Pedro) de la que surge un tercer conjunto o conjunto diferencia (las 3 canicas que Pedro tiene menos que Juan). Dicho de otro modo, en los problemas de comparación se pueden distinguir tres conjuntos: un conjunto comparado (siguiendo con el ejemplo, las canicas que posee Pedro) que se pone en relación (en términos comparativos del tipo "más/menos que", "mayor/menor 
que"...) con un conjunto referencia (las canicas que tiene Juan), dando lugar esta comparación a un tercer conjunto, la diferencia (en este caso, 3 canicas). De acuerdo con estos modelos, cuando los niños se enfrentan a la resolución de este tipo de problemas necesariamente han de comprender los términos lingüísticos en los que está formulado el problema, interpretar la situación planteada, elegir la operación aritmética adecuada a esa situación y ejecutar los cálculos necesarios para llegar a solución del problema.

Sin embargo, de acuerdo con algunos modelos (e.g., Riley et al., 1983; Riley y Greeno, 1988) para resolver los problemas más difíciles (en el caso de los problemas de comparación, aquellos en los que el conjunto desconocido es el conjunto referencia, por ejemplo, "Juan tiene 15 caramelos, y tiene 12 más que Pedro. ¿Cuántos caramelos tiene Pedro?”) la construcción de esta representación semántica no es suficiente, sino que es necesario aplicar el conocimiento esquemático parte-todo sobre la información proporcionada.. Dicho de otro modo, la información original del problema debe re-representarse en términos de la estructura parte-todo subyacente a la situación planteada, de tal forma que en la comparación cuantitativa el alumno ha de saber que "conjunto menor = conjunto mayor - conjunto diferencia" (en el ejemplo, "caramelos de Pedro = caramelos de Juan -12") o que "conjunto mayor = conjunto menor + conjunto diferencia" (Stern, 1993) (esto es, "caramelos de Juan = caramelos de Pedro + 12), lo cual permite inferir desde la frase relacional del problema si el conjunto mencionado en la frase de asignación es el conjunto mayor o el conjunto menor y, en función de esta inferencia, decidir qué operación es necesaria para resolver el problema (sumar o restar). De este modo, este conocimiento parte-todo permite transformar directamente la información textual en una ecuación matemática.

Además de la representación en términos de la estructura parte-todo, Lewis y Mayer (1987) propusieron una segunda estrategia para resolver los problemas de comparación más difíciles (los problemas con el conjunto referencia desconocido), denominada reestructuración lingüística. Esta estrategia permite transformar este tipo de problemas en problemas de comparación más fáciles transformando la frase relacional del problema. Siguiendo con el ejemplo que propusimos unas líneas más arriba, la estrategia de reestructuración lingüística consistiría en modificar la frase relacional para que el referente no sea el conjunto de caramelos que tiene Pedro, que se desconoce, sino los 15 caramelos que tiene Juan, de manera que la frase relacional dejaría de ser "(Juan) tiene 12 caramelos más que Pedro" para transformarse en "Pedro tiene 12 caramelos menos que Juan”.

Por último, algunos modelos han señalado la importancia de la comprensión de la información situacional en el proceso de resolución de problemas (Kintsch, 1988, 1998; Nathan, Kintsch y Young, 1992; Reusser, 1988; Staub y Reusser, 1995), entendiendo por información situacional toda aquella referente a los aspectos cualitativos del problema en términos de la estructura intencional, temporal y causal de la situación propuesta.

En resumen, para interpretar qué es lo que ocurre en la interacción que tiene lugar entre profesores y alumnos hemos utilizado este conocimiento sobre los procesos cognitivos implicados en la resolución de problemas.

\section{¿CÓMO AYUDAN LOS PROFESORES A SUS ALUMNOS A RESOLVER PROBLEMAS ARITMÉTICOS?}

Para contestar esta pregunta, hemos realizado varios trabajos en los que comparamos las interacciones verbales que mantuvieron un grupo de profesores con sus alumnos. Por ejemplo, en uno de estos estudios comparamos a 3 profesores 
en ejercicio (con más de 10 años de experiencia docente) con 3 futuros profesores (estudiantes del último año de Magisterio), cuando resolvieron conjuntamente con sus alumnos (de entre 8 y 10 años) un problema aritmético (Rosales, Orrantia, Vicente y Chamoso, 2008).

\section{¿Qué problemas hemos utilizado en estos estudios?}

En estos estudios, les propusimos a los profesores que resolvieran conjuntamente con sus alumnos el siguiente problema:

Un bodeguero quiere renovar las cubas de vino porque este año ha comprado más uvas. En las cubas de madera que tiene caben 158 litros de vino. Pero en estas cubas de madera caben 26 litros menos que en unas nuevas cubas metálicas. ¿Cuántos litros de vino caben en una cuba metálica?

De acuerdo con los modelos de resolución de problemas que hemos descrito en la primera parte de este trabajo, este problema plantea la comparación entre dos conjuntos. Para resolverlo es necesario identificar el conjunto de referencia, el conjunto comparado y el conjunto diferencia. De forma más concreta, en primer lugar, la frase de asignación ("En las cubas de madera que tiene caben 158 litros de vino") especifica el valor numérico del conjunto de comparación. En segundo lugar, la frase relacional ("en estas cubas de madera caben 26 litros menos que en unas nuevas cubas metálicas") establece la cantidad del conjunto referencia en función del conjunto comparado a través de la diferencia conocida entre ambos conjuntos. Por ultimo, la pregunta del problema hace referencia al valor desconocido del conjunto de referencia ("¿Cuántos litros de vino caben en una cuba metálica?").

Dado que este problema pertenece a una de las categorías más difíciles de problemas de comparación, siguiendo los modelos teóricos expuestos anteriormente, para poder resolver este problema es necesario proyectar la información textual en el esquema parte-todo subyacente al mismo. De esta manera, el alumno debe inferir de la frase relacional del problema que dado, por un lado, que el conjunto comparado (las cubas de madera) es el conjunto menor y que el conjunto referencia (las cubas de metal) es el mayor, y por otro lado, que se conoce la diferencia entre ambos, el problema puede resolverse con la operación $158+26$ ya que "conjunto mayor $=$ conjunto menor + diferencia). Otro modo de resolver este problema es a través de la estrategia de reestructuración lingüística (Lewis y Mayer, 1987), de manera que la frase relacional de este problema de comparación con conjunto referencia desconocido ("en estas cubas de madera caben 26 litros menos que en unas nuevas cubas metálicas") puede transformarse en "en las cubas de metal entran 26 litros más que en las de madera”. De esta manera la información proporcionada por la frase relacional de manera que el término "más que" coincide con la operación necesaria para resolver el problema, en este caso una suma.

Por último, para comprender el contexto situacional, el alumno ha de inferir las necesidades del bodeguero y las intenciones que de éstas se derivan, de las cuales se extrae la estructura causal del problema. Esta representación cualitativa debería incluir las ideas de que el bodeguero tenía muchas uvas, y que debido a esto produciría más vino y por esta razón necesita comprar unas cubas nuevas más grandes.

De manera gráfica esto se puede representar del siguiente modo (ver Figura 1).

\section{¿Cómo hemos analizado la interacción?}

Para el análisis de cada una de las interacciones que mantuvieron profesor y alumnos para resolver este problema, utilizamos una variante de un Siste- 
FIGURA 1

Representación esquemática de los procesos cognitivos implicados en la resolución de un problema de comparación. Adaptado de Rosales et al. (2008)

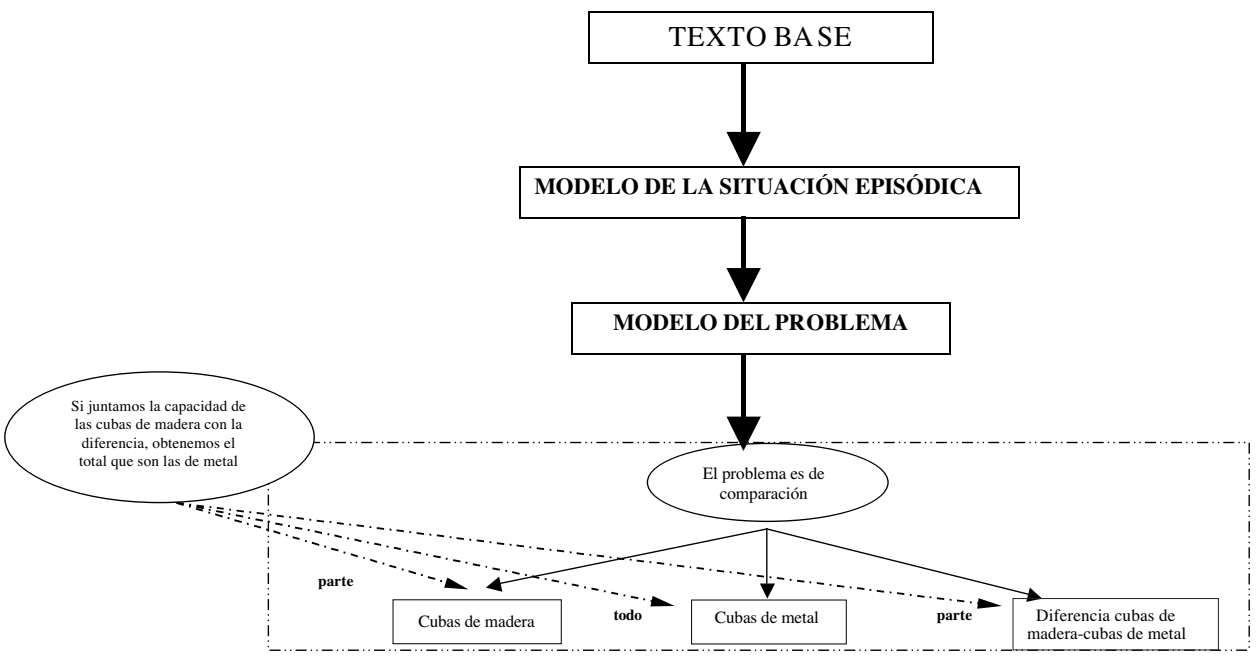

ma de Análisis que ya habíamos empleado en otras ocasiones. En concreto, una versión de este Sistema fue usado para estudiar las interacciones entre profesores y alumnos cuando leen conjuntamente un texto (Sánchez, Rosales y Suárez, 1999; Rosales, Iturra, Sánchez y de Sixte, 2006), o entre profesores y alumnos cuando tratan de resolver problemas aritméticos (del Río, Sánchez y García, 2000; Rosales et al., 2008). La última versión del Sistema de Análisis (Sánchez et al., 2008) permite responder a tres cuestiones básicas sobre la interacción:

a) ¿Cómo es el contexto que se crea para el desarrollo de la tarea? O lo que es lo mismo: ¿cómo se canaliza la interacción de profesores y alumnos? En este caso, el Sistema de Análisis nos permite tomar como referencia diferentes unidades de análisis. De mayor a menor serían: Unidad Didáctica, Sesión, Actividad Típica de Aula, Episodio y Ciclo de interacción.

b) ¿Qué contenidos y procesos surgen de la interacción? De otra manera: ¿cuáles son los procesos y contenidos relacionados con la resolución de la tarea que ponen en marcha durante la interacción? En este caso, el Sistema de Análisis permite identificar cuáles son los contenidos que se hacen públicos durante la interacción en términos de ideas o proposiciones que se llegan a compartir entre profesor y alumnos.

c) ¿Quién es el responsable de la puesta en marcha de esos procesos y contenidos? De otro modo: ¿cuál es el nivel de participación de profesor y alumnos en el desarrollo de procesos y contenidos?

\section{¿Qué resultados hemos encontrado?}

A continuación presentamos algunos de los resultados más interesantes que hemos encontrado en nuestros estudios. Para ello, trataremos de contestar a cada una de las preguntas a que acabamos de hacer referencia. En concreto, ¿cómo es el contexto que se crea para el desarrollo de la tarea?, ¿qué contenidos y procesos surgen de la interacción? y, por último, ¿quién es el responsable de la puesta en marcha de esos procesos y contenidos? 
¿Cómo es el contexto que se crea para la resolución de problemas aritméticos en el aula?

Para dar respuesta a esta pregunta hemos tomando como unidad de análisis más amplia las Actividades Típicas de Aula o ATAs. El término Actividad Típica de Aula ha sido utilizado por Jay Lemke para referirse a las actividades que se repiten con una cierta frecuencia en las aulas; la revisión de deberes, el trabajo individual en el pupitre o la explicación del profesor (Lemke, 1997) y es semejante a los Formatos Instruccionales empleados por Susan Stodolsky (Stodolsky, 1992).

En la tabla que presentamos a continuación se muestran dos ejemplos de ATAs $^{2}$ de resolución de problemas. Una de ellas corresponde a un profesor en ejercicio y la otra a un futuro profesor y están tomadas de nuestra propia muestra. Ambas ATAs nos dan una idea de cómo es el tipo de contexto de interacción que creó cada profesor.

TABLA I

Actividades Típicas de Aula de un profesor en ejercicio y un profesor en formación

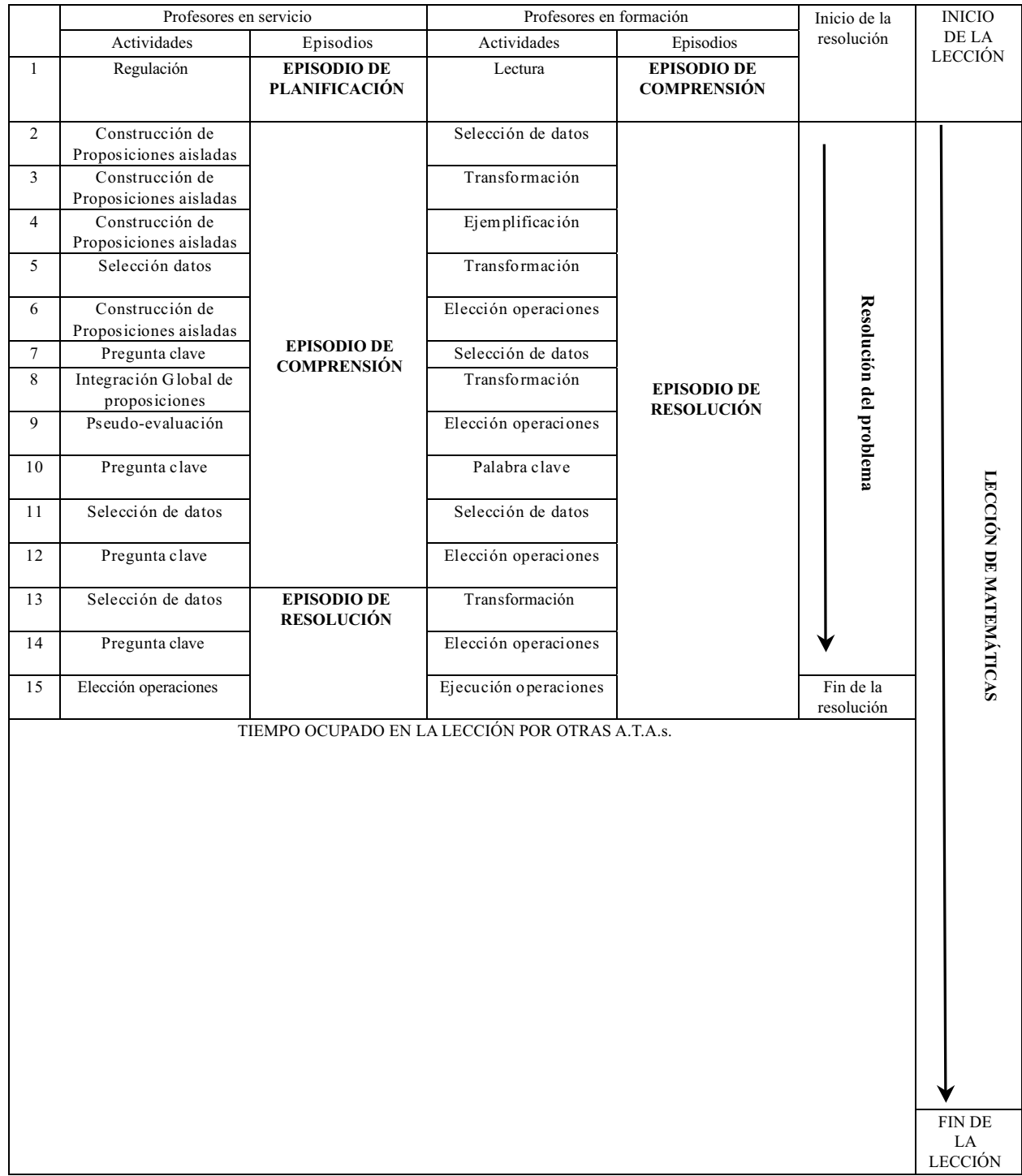


Como vemos, las ATAs de resolución de problemas agrupan un conjunto de actividades o Episodios que, de forma regular, se van sucediendo con unos objetivos y planes de trabajo específicos y que, en última instancia, son compartidos por profesor y alumnos. Básicamente, la estructura formal de ambas ATAs es la misma. Se comienza con la lectura del problema, seguidamente, toda la actividad se dirige a la comprensión del problema y, por último, se aplica la operación necesaria para resolverlo. En ambos casos, el patrón que se desprende sería algo parecido a: Resolver un problema requiere leerlo correctamente, pensar qué tenemos que hacer para solucionarlo correctamente y, por último, decidir la operación y realizarla.

Indudablemente, cabría la posibilidad de considerar otras estructuras formales alternativas o complementarias que dieran entrada a patrones de participación diferentes. Por ejemplo, podemos imaginar al profesor dedicando un espacio del tiempo a planificar y explicar la relevancia de la resolución de problemas o su sentido dentro de la Unidad Didáctica en la que están inmersos. En estos casos, el patrón sería algo diferente: Tenemos que entender qué sentido tiene el que todos los días dediquemos un tiempo a hacer problemas...

En cualquier caso, merece la pena reseñar una diferencia entre los Episodios incluidos en cada una de las ATAs y que resulta evidente a los ojos del lector. Efectivamente, el número de Ciclos de interacción, o de otro modo, el tiempo que dedican un profesor y otro a cada una de las actividades no es el mismo. En un caso, el del profesor en ejercicio, los Episodios de Comprensión son más extensos que los Episodios de Resolución, mientras que en el otro, el del profesor en formación, ocurre lo contrario. Esto es, el Episodio de Resolución comprende un número mucho mayor de Ciclos de interacción que el Episodio de Comprensión. ¿Qué quiere decir esto? En principio, este comportamiento nos podría estar indicando que uno de los profesores está muy preocupado porque sus alumnos comprendan el problema, mientras que el otro profesor no lo está tanto.

De todas formas, sólo podremos saber si estamos en lo cierto si realizamos un análisis más detallado de qué es lo que hacen durante cada uno de los Episodios. Esto supone que nuestra mirada debe dirigirse a los Ciclos de Interacción como unidad de análisis y, concretamente, a los contenidos que se hacen públicos durante la interacción:

\section{¿Qué contenidos y procesos surgen durante la resolución de problemas aritméticos en el aula?}

Para estudiar qué se hace público durante la interacción o, si se quiere de otra manera, sobre qué hablan profesor y alumnos durante la interacción, hemos estudiado detenidamente el discurso de profesores y alumnos. En concreto, para cada Episodio hemos aislado los Ciclo de interacción que lo componen y, seguidamente, hemos identificado las ideas desarrolladas así como los procesos implicados en la resolución de problemas a los que hicieron referencia. Para clarificar esta cuestión a continuación mostramos un ejemplo (ver Tabla II).

En definitiva, actuando de este modo, podemos identificar qué es lo que se hace público durante la interacción y, lo que es más importante, podemos interpretar de qué hablan profesor y alumnos a partir de lo que significa resolver un problema matemático.

Cuando tomamos en consideración este dato, los resultados nos muestran que ambos grupos de competencia (profesores en servicio y profesores en formación) hacen público el contenido necesario para la resolución del problema. Concretamente, todos los profesores que hemos estudiado identificaron los conjuntos implicados en el problema, esto es, el conjunto referencia, el conjunto comparación y el conjunto diferencia. Del mismo modo, todos los profesores hicieron 
La resolución de problemas aritméticos en el aula / J. Rosales et al.

TABLA II

Ejemplo de aplicación del sistema de análisis a un fragmento de interacción correspondiente al episodio de comprensión de un profesor en servicio

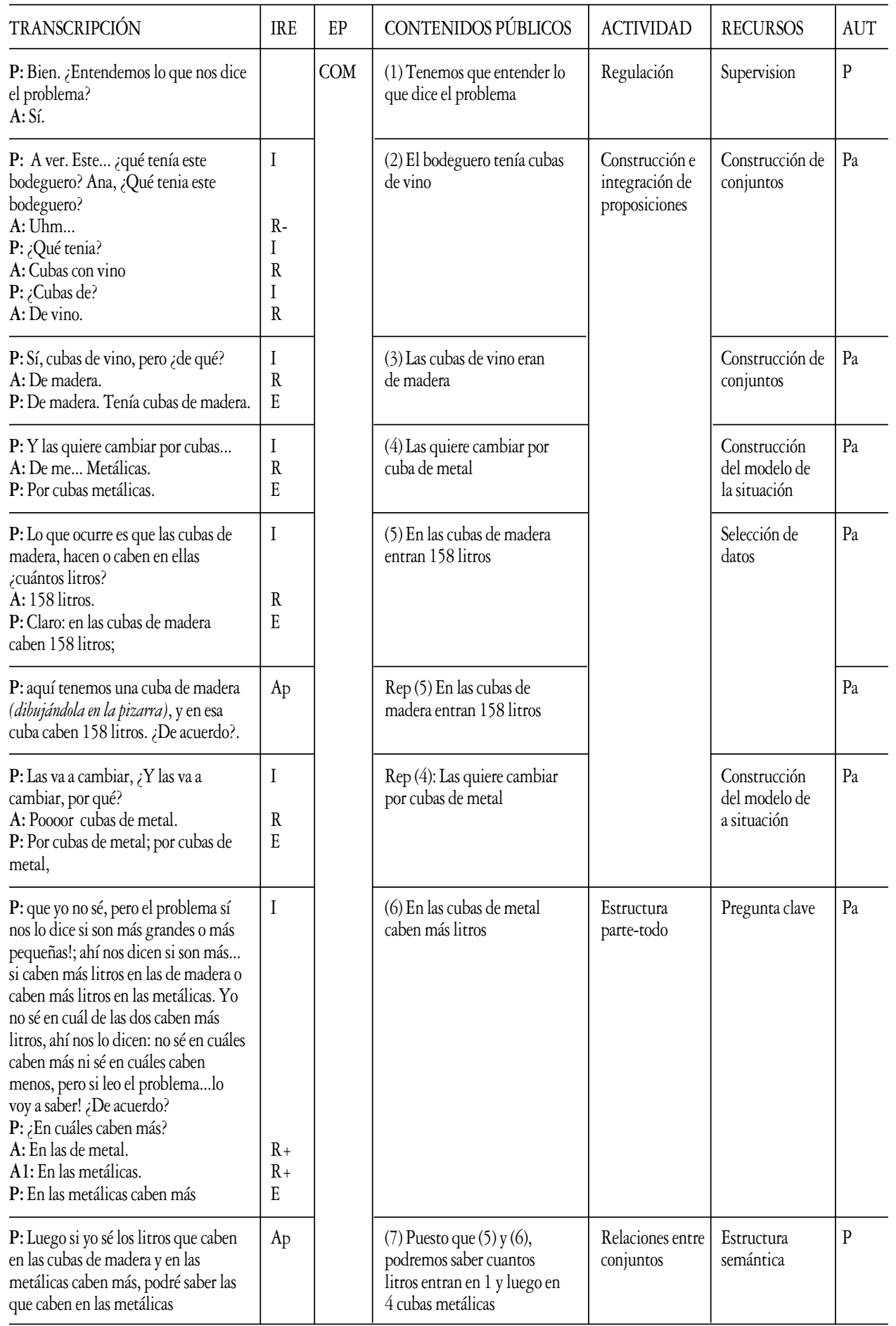


público el esquema parte-todo y parte de ellos hicieron mención al Modelo de la Situación que el problema evoca. En la siguiente figura se clarifica esta cuestión presentando la media de Ciclos de interacción que desarrollaron cada grupo de competencia a cada una de las actividades implicadas en la resolución de problemas. En la figura 2 se diferencia entre las actividades dedicadas a la comprensión del tema de las actividades dedicadas a la resolución.

FiguRA 2

Media de ciclos dedicados por los profesores en servicio y en formación a cada actividad del proceso de resolución

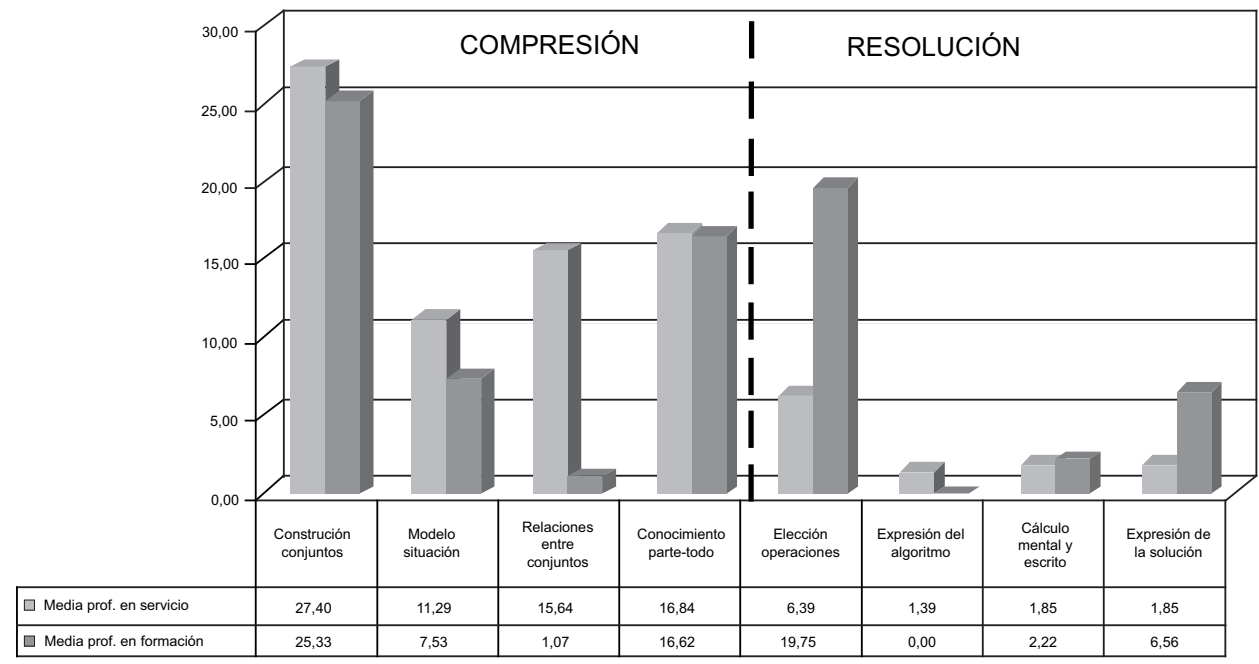

El que ambos grupos de competencia hagan público un contenido similar podemos interpretarlo desde los propios modelos cognitivos sobre resolución de problemas. Así, sabemos que si queremos resolver de forma satisfactoria el problema, debemos hacer explícita la estructura comparativa del problema e identificar su estructura parte-todo. En nuestro caso: en las cubas de madera que tiene caben 158 litros de vino (conjunto comparación), en estas cubas de madera caben 26 litros menos que en unas nuevas cubas metálicas (conjunto referencia) y, por último, si en las cubas de madera caben menos litros, en las de metal cabrán más litros

Hay, sin embargo, algunas diferencias que afectan al comportamiento de ambos grupos de profesores en cada uno de los Episodios. Así, en el Episodio de Comprensión existen claras diferencias entre el porcentaje de ciclos dedicados a la identificación de relaciones entre conjuntos así como a la creación de un Modelo de la Situación. En concreto, los datos nos dicen que los profesores en ejercicio, además de construir conjuntos, hacen ver a sus alumnos qué relaciones se establecen entre esos conjuntos. Por ejemplo.

Profesor: de manera que si sabes cuántos litros caben en las cubas de madera, y en las de metal caben 26 más, puedo saber cuántos litros caben en una cuba de metal.

En el ejemplo, el profesor hace explícito a sus alumnos que existe una relación entre las barricas de madera y las de metal y que si se tienen en cuenta ambos datos podremos saber en cuál de las dos cabrá más.

Además, aunque en este caso las diferencias no son tan claras, los profesores en ejercicio tratan de recrear a sus alumnos el contexto situacional de la información que aparece en el problema. Por ejemplo. 
Profesor: Mirad: este bodeguero es un señor que tiene muuuchas muchas vides, ;luego tiene una gran viña!, y quiere renovar las cubas de vino porque este año él ha comprado más uvas de lo que habitualmente compra.

Este fragmento es un buen ejemplo de cómo el profesor trata de explicitar la estructura causal de los diferentes eventos que tienen lugar en el transcurso de la situación descrita en el problema para, posteriormente, utilizar esa estructura causal para establecer las relaciones entre los conjuntos y también entre las cantidades. Reusser (1988) afirma que el Modelo de la Situación Episódica representa la estructura intencional, temporal y causal de la situación que se describe en el problema, y que la generación de este modelo es un paso imprescindible en el proceso de resolución, de modo que únicamente cuando el alumno ha generado este modelo estará en condiciones de establecer las relaciones matemáticas existentes entre los conjuntos y resolver el problema.

Por otra parte, en el caso del Episodio de Resolución, son los profesores en formación los que dedican un porcentaje diferente de ciclos a la selección del algoritmo así como a la expresión del resultado. Veamos algún ejemplo:

Profesor: A ver, Sara, dime qué tendríamos que hacer en el problema.

Alumna 1: Pues tendremos que saber cuántos litros entran en la ...en las nuevas cubas metálicas.

Profesor: Entonces, ¿qué tendríamos que hacer?

Alumna 1: Sumarlo.

Alumno 2: 158 más 26.

En este caso, el profesor anima a sus alumnos a decidir sobre la operación que se tendrá que utilizar para resolver el problema. La preocupación, por tanto, es elegir bien la operación.

Lo mismo podríamos decir de la expresión de la solución. Los profesores en formación dedican una mayor proporción de ciclos a expresar correctamente el resultado de la operación

Profesor: ¿Y quéson éstos?

Alumno: Eh... Litros que caben en una cuba de metal

Profesor: Litros que caben en una cuba de metal, en una de metal.

En definitiva, a partir de estos resultados podemos afirmar que para el grupo de profesores que hemos estudiado, los profesores en ejercicio dedicaron un mayor porcentaje de ciclos a clarificar cuestiones relacionadas con la comprensión del problema, mientras que los profesores en formación estuvieron más pendientes de las cuestiones relativas a su resolución.

De la misma forma, si consideramos de forma detallada cómo se distribuyeron cada una de las actividades a lo largo de los Episodios de Comprensión y de Resolución podemos observar claras diferencias. Veamos la figura 3 en la que se muestra el porcentaje de Ciclos de interacción desarrollados en cada Episodio.

En la figura 3 podemos comprobar que los profesores en ejercicio incluyeron un buen número de Ciclos de interacción en el Episodio de Comprensión. Esto quiere decir que estos profesores dedicaron la mayor parte de la interacción con sus alumnos a crear una representación coherente del problema. Por el contrario, el tiempo dedicado a la resolución del problema fue mucho menor, si consideramos en porcentaje de ciclos incluidos en el Episodio de Resolución. Por su parte, los profesores en formación incluyeron la mayor parte de sus Ciclos de interacción en el Episodio dedicado a la Resolución por lo que dedicaron la mayor parte del tiempo a la resolución del algoritmo necesario para resolver el problema.

Igualmente, si consideramos las actividades relacionadas directamente con la comprensión del problema, podemos comprobar que los profesores en ejercicio localizan todas ellas en el Episodio de Comprensión, mientras que 
FIGURA 3

Porcentajes medios de ciclos dedicados por profesores en servicio y en formación a actividades de comprensión y de resolución

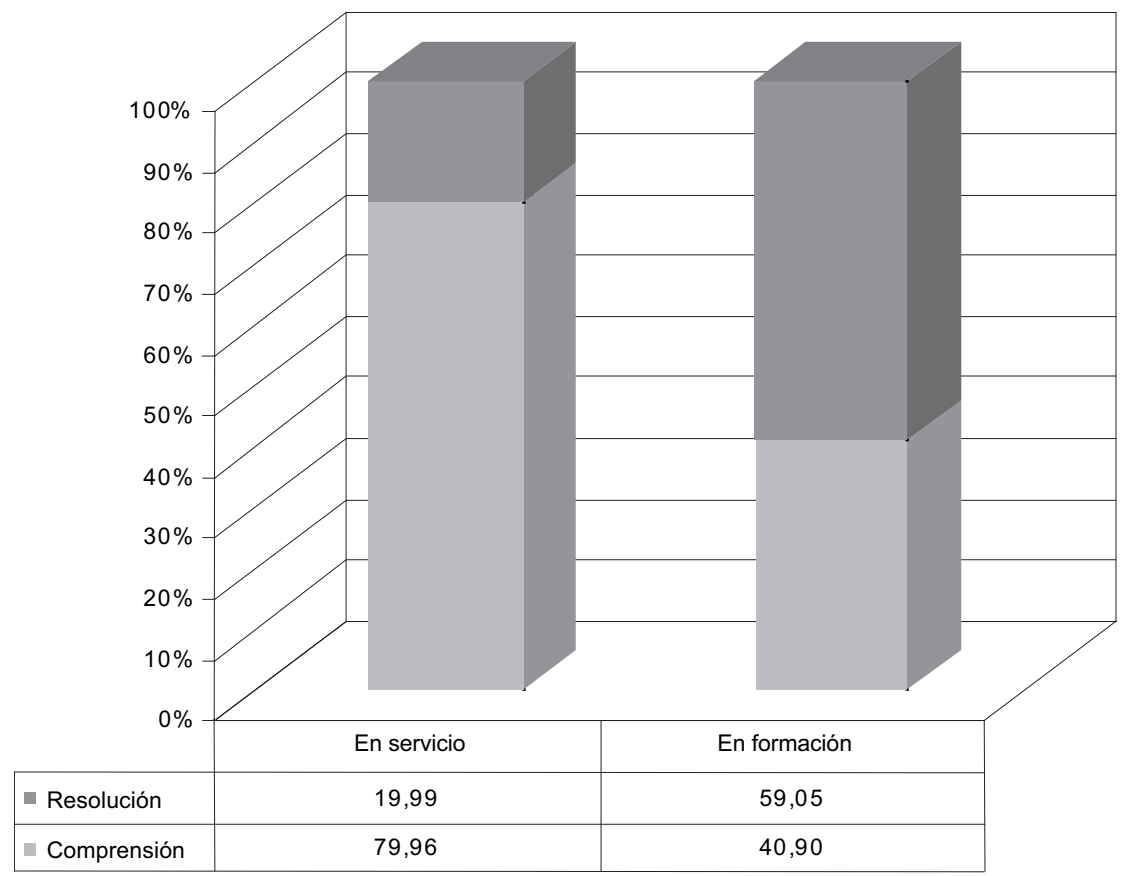

los profesores en formación lo hacen en el Episodio de Resolución. Esto quiere decir que los primeros se diferenciaron de los segundos en que, antes de la resolución del problema, garantizaron la comprensión de todos los elementos necesarios para la resolución del problema: construcción y relación entre conjuntos e identificación de la estructura parte-todo. Por su parte, los profesores en formación, localizaron la mayoría de las actividades relacionadas con la comprensión en el Episodio de Resolución. Con toda seguridad, esto se debió a que sus alumnos cometieron un número mayor de errores en la selección de la operación y en la expresión del resultado, lo que les obligó a reiniciar de nuevo la lectura del problema así como su comprensión. Seguramente, por esto mismo podemos observar un número mayor de Ciclos de interacción dedicados a la selección del algoritmo.

En resumen, estos resultados nos permiten concluir que los profesores en ejercicio consiguieron crear con sus alumnos una comprensión compartida con sus alumnos sobre los elementos esenciales implicados en la comprensión del problema antes de tomar una decisión sobre la operación que se debería llevar a cabo para su resolución. Por el contrario, los profesores en formación sólo consideraron esta cuestión cuando constataban que sus alumnos fracasaban en la elección de la operación. Por tanto, el comportamiento estratégico de los profesores en ejercicio frente a los profesores en formación consistió en que los primeros garantizaron una comprensión conjunta del problema antes de tomar una decisión sobre el signo de la operación a realizar.

\section{¿Quién es el responsable de la puesta en marcha de esos procesos y contenidos?}

Por último, para identificar cuál es el papel que juegan profesor y alumnos durante la interacción hemos cuantificado qué parte de responsabilidad asumie- 
ron cada un de ellos a lo largo de la interacción. Para ello, hemos utilizado una escala en la que representamos:

(P) La construcción de las ideas es asumida de forma autónoma por el profesor sin la participación de sus alumnos:

Profesor: Un momento, un momento que lo primero que vamos a hacer es comprender la lectura de este problema. ¿eh?. Qué me dicen? , qué conozco?, quétengo que averiguar?, eh?

(A) La construcción de las ideas es asumida de forma autónoma por los alumnos sin ninguna ayuda de sus profesores. En nuestro estudio no encontramos ningún ejemplo de autonomía completa del alumno en ningún ciclo, ya que en todos ellos el profesor participaba en algún grado.

(Pa) La construcción de las ideas es asumida de forma conjunta aunque con una mayor participación del profesor, que es el que toma una mayor responsabilidad en la dirección de la interacción:

Profesor: Lo primero, vamos a empezar con la pregunta, Miguel.

¿Qué te dice la pregunta del problema?

A: Que averigüiemos cuántos litros entran en una cuba...

A1: Metálica

P: Metálica.

(Ap) La construcción de las ideas es asumida de forma conjunta aunque con una mayor participación del alumno, que es quien toma una mayor responsabilidad en la dirección de la interacción:

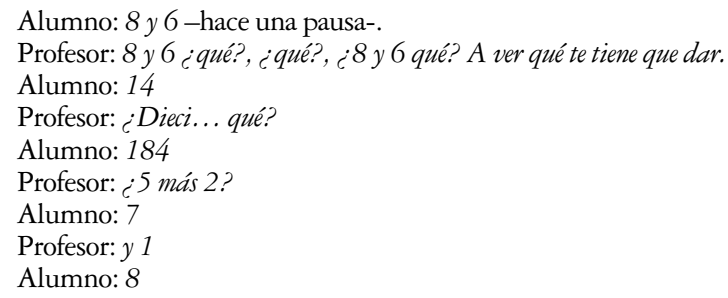

Utilizando esta escala, una de las medidas que hemos considerado en nuestros estudios ha sido el porcentaje de Ciclos de interacción que se dedicó cada grupo a diferentes actividades implicadas en la resolución de problemas. Por ejemplo, en la tabla III presentamos algunos de los resultados obtenidos actuando de este modo.

La figura 4 muestra que, los alumnos que mantuvieron la interacción con profesores en ejercicio, asumieron una mayor responsabilidad en la resolución de la tarea. En concreto, los profesores asumieron un mayor control en la construcción de explicitación de las relaciones entre conjuntos, dejando a sus alumnos una mayor cuota de responsabilidad en la construcción de conjuntos, en la creación de la estructura parte-todo así como en la elaboración de un modelo de la situación para el problema. En la siguiente tabla presentamos ejemplos que ilustran estos resultados.

Por su parte, si consideramos la figura 5 veremos cómo se comportaron los profesores en formación y sus alumnos.

En este caso, los alumnos tuvieron una participación mucho menos relevante ya que, prácticamente, en todas las actividades consideradas toda la responsabilidad recayó sobre el profesor. Quizá el ejemplo más significativo sea que mientras que los profesores en servicio suscitaban un mayor nivel de participación de los alumnos al acceder a la estructura parte-todo del problema, los profesores en for- 
FIGURA 4

Grado de participación de profesores en servicio y sus alumnos en actividades del episodio de comprensión

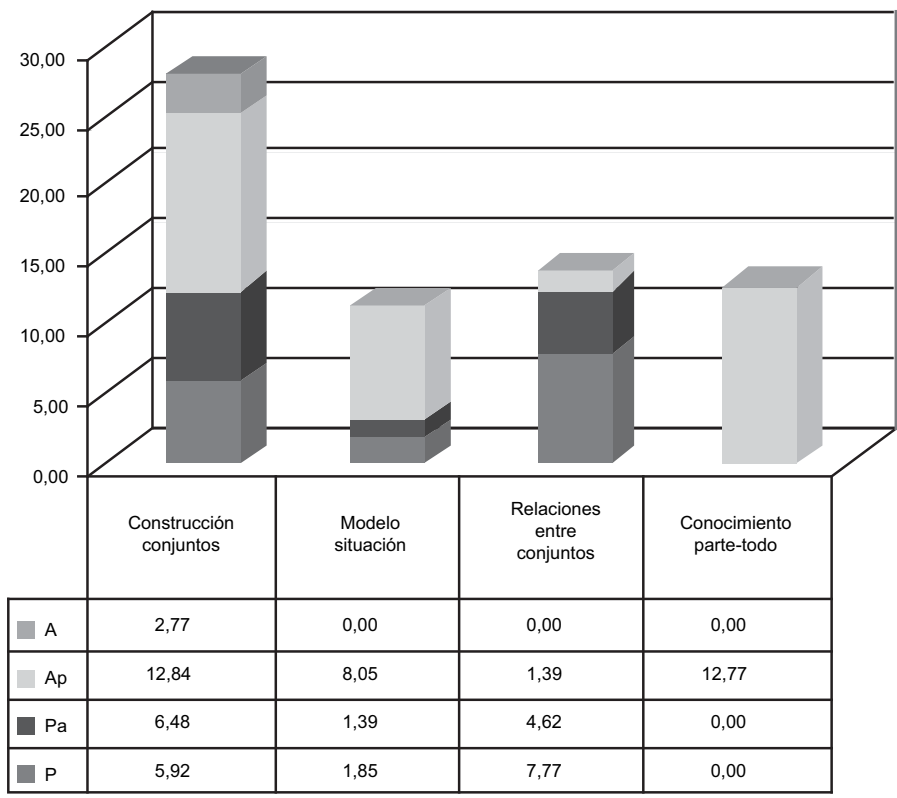

TABLA III

Ejemplos de grado de participación de de profesores en servicio y sus alumnos en actividades del episodio de comprensión

\begin{tabular}{llll}
\hline \multicolumn{1}{c}{$\begin{array}{c}\text { Relaciones entre } \\
\text { conjuntos }\end{array}$} & \multicolumn{1}{c}{$\begin{array}{c}\text { Construcción de } \\
\text { conjuntos }\end{array}$} & $\begin{array}{c}\text { Estructura parte- } \\
\text { todo }\end{array}$ & $\begin{array}{c}\text { Modelo de la } \\
\text { situación }\end{array}$ \\
\hline P: Luego si yo sé los & P: Muy bien. ¿Qué & P: Pero, ¿qué ocurre, & P: Bien. ¿Cuál era el \\
litros que caben en las & sabemos de la cuba de \\
cubas de madera y en & madera, señorita Isabel? & $\begin{array}{l}\text { comparándolas con la } \\
\text { de metal... Isabel? }\end{array}$ & $\begin{array}{l}\text { problema del } \\
\text { bodeguero? }\end{array}$ \\
las metálicas caben & A: Que entran 158 litros & $\begin{array}{l}\text { A: Que la de metal } \\
\text { tienen 26 litros menos. }\end{array}$ & $\begin{array}{l}\text { A: Que no le entraba el } \\
\text { vino porque había }\end{array}$ \\
más, podré saber las & P: ...158 litros. & P: MEENOS!, iqué quíén? & comprado más uvas. \\
que caben en las & Lo escribimos aquí: en & A: Que la de madera. Uhm... & P: Había comprado \\
metálicas; y cuando ya & las cubas de madera & P: A ver, piensa. & más uvas, y... \\
sepa lo que caben en & entra 158 litros. & ¿En quién entran más, & A: No le entraba el \\
una cuba podré saber lo & & en la de madera o en la & vino. \\
que caben... en cuatro! & & de metal? ¿Carlos? & P: No le entraba el \\
& & A: En las de Metal & vino en las \\
& & P: En las de Metal. & A: Cubas, cubas \\
& & & P: CUBAS que tenía, \\
& & & iverdad que sí? \\
\hline
\end{tabular}

mación realizaban la transformación de la frase relacional sin que el alumno tuviera participación alguna:

P: Si en las cubas de madera caben 26 litros menos que en las de metal, en las de metal, aquí, aquí, obviamente, cabrán 26 litros más que en la de madera ¿no?

A $4:$ Si

Como vemos en este ejemplo, la única participación que tiene el alumno en el acceso a la estructura parte-todo del problema es un "Sî" meramente testimonial 


\section{FIGURA 5}

Grado de participación de profesores en formación y sus alumnos en actividades del episodio de comprensión

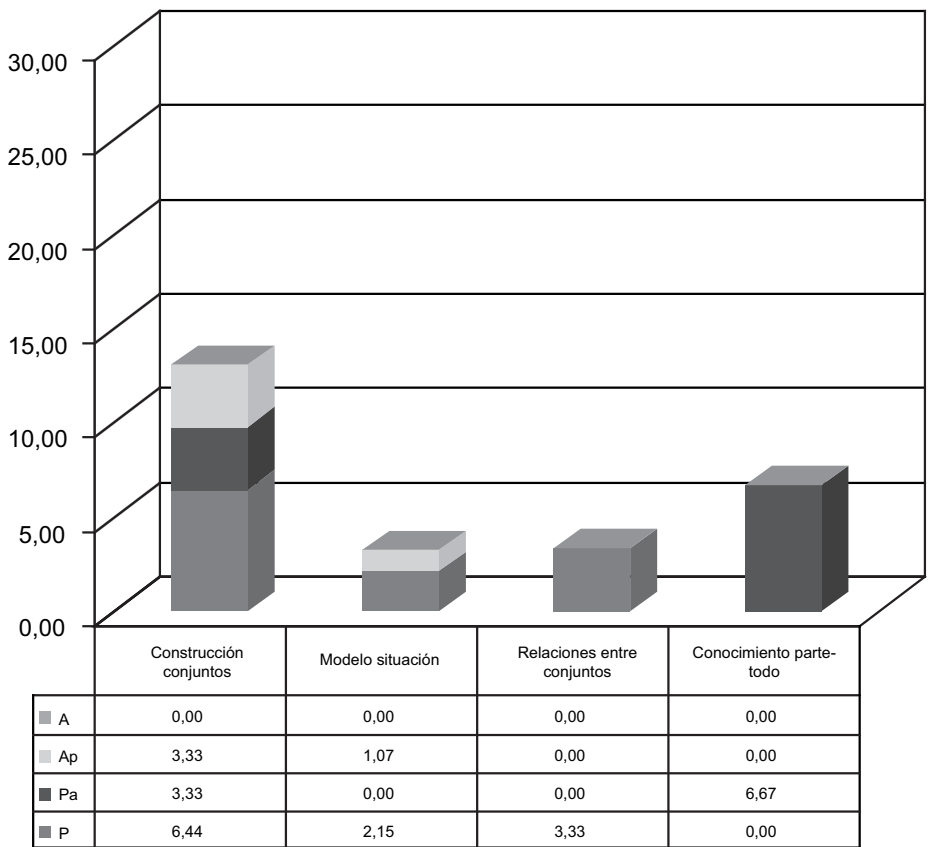

a un requerimiento por parte del profesor que no va más allá de ser una pregunta retórica, ya que es el profesor quien hace todo el razonamiento y el alumno se limita a asentir.

En resumen, nuestros resultados nos sugieren que los profesores en ejercicio dieron a sus alumnos un número mayor de oportunidades para incorporarse a la resolución efectiva de los problemas. De forma más específica, podemos decir que este grupo de profesores permitió a sus alumnos asumir una mayor responsabilidad en la elaboración del contenido público. Por el contrario, los profesores en formación apenas dejaron espacio para que sus alumnos colaborasen en la elaboración de la información ya que ellos mismos asumieron esta responsabilidad.

\section{CONCLUSIÓN}

En este trabajo hemos presentado algunos de los resultados más sobresalientes que hemos encontrado cuando hemos estudiado la interacción profesor-alumnos mientras resuelven conjuntamente un problema aritmético. En concreto, nuestro objetivo ha sido mostrar en qué medida el estudio de la interacción se puede enriquecer cuando se realiza en consonancia con el conocimiento sobre lo que implica la resolución de un problema. Puesto que disponemos de modelos cognitivos, suficientemente contrastados, de cómo se comporta nuestra mente cuando resuelve un problema, parece una falta de coherencia aplicarse al estudio de cómo se promociona la resolución de problemas en un contexto educativo, sin tener presente todo este conocimiento disponible.

En nuestro caso, después de analizar los resultados obtenidos, hemos podido comprobar cómo los profesores en ejercicio se comportaron de un modo mucho más estratégico que los profesores en formación. De una forma más específica, los primeros trabajan en el aula para conseguir que sus alumnos comprendan el 
contenido semántico que subyace a cada problema antes de tomar una decisión sobre la operación a utilizar. Por el contrario, el grupo de profesores en formación, consideran la necesidad de abordar la comprensión del problema, únicamente cuando los alumnos se equivocan en la selección de la operación. En consonancia con la revisión de Hogan et al. (2003) el primer grupo de profesores se comportó de un modo mucho más eficiente, usando estrategias más adaptadas a la dificultad de la tarea.

Ahora bien, más allá de constatar estas diferencias podemos ahondar más en ellas si consideramos la naturaleza estratégica de las ayudas en términos de las demandas cognitivas de la tarea. En este sentido, este conocimiento nos ha permitido reinterpretar la tarea del profesores en términos de cuáles de estos procesos son los que se promocionan en una determinada interacción. Así, podríamos afirmar que no todas las actividades que el profesor desarrolla en el aula tienen el mismo valor a la hora de resolver un problema.

Por otra parte, en lo que se refiere a la autonomía que asumieron profesores y alumnos en la resolución de la tarea, nuestros datos se corresponden con los obtenidos por Blanton, Berenson y Norwood (2001), quienes encontraron cómo los profesores sin experiencia suelen ser menos dialógicos que sus pares de experiencia superior. En cualquier caso, lo que nuestro estudio aporta a trabajos como éste es que nos ayuda a entender en qué actividades el profesor es más o menos dialógico y en qué otras debería o no debería serlo. O lo que es lo mismo, al considerar la responsabilidad de los participantes en la interacción a partir de modelos cognitivos, podemos identificar en qué momentos específicos de la resolución del problema fueron más dialógicos. Si se quiere, esto mismo se puede decir de otro modo. Lo verdaderamente importante no es si un profesor es más o menos dialógico con sus alumnos sino si ese diálogo se establece en torno a los elementos esenciales de la tarea.

Evidentemente, estas conclusiones deben interpretarse teniendo en cuenta la muestra con la que han sido obtenidos nuestros resultados. En primer lugar, han sido, únicamente, 6 los profesores estudiados, por tanto, en futuros estudios será necesario aumentar la muestra de profesores para poder presentar nuestras conclusiones con una mayor contundencia. En segundo lugar, el hecho de trabajar, únicamente, con un tipo de problema, hace que debamos limitar las conclusiones a las interacciones que se mantienen con este tipo de problemas. En cualquier caso, en futuros estudios habrá que considerar la presencia de tipos de problemas diferentes.

\section{Notas}

${ }^{1}$ Cuando se toma como punto de partida la noción de buen aprendizaje y buena enseñanza se interpreta que estamos ante un
proceso que contiene al menos cuatro propiedades: a) debe entenderse desde las reglas de la comunicación humana y, por
tanto, con el objetivo de compartir significados b) tiene una naturaleza colaborativa, en el sentido que las partes (alumnos y
profesores) deben tener algún grado de participación activa c) tiene una naturaleza dinámica, en el sentido de que, una de las
partes (el alumno) habrá de incrementar su contribución según avanza el proceso, y d) su resultado deseable es una compren-
sión profunda y sustantiva del material (Sánchez y Rosales, 2005)
${ }^{2}$ Cada una de estas ATAs se desarrollaron en sendas Unidades Didácticas de Matemáticas y, además, estuvieron incluidas en
sesiones específicas que se llevaron a cabo en dos clases de 3er curso de Educación Primaria.

\section{Referencias}

BRIARS, D. J. \& LARKIN, J. H. (1984). An integrated model of skill in solving elementary word problems. Cognition and Instruction, 1, 245-296.

Blanton, M. L., Berenson, S. B. \& NorwOOD, K. (2001). Using classroom discourse to understand a prospective mathematics teacher's developing practice. Teaching and Teacher Education, 17, 227-242.

DEL Río, I., SÁNCHEZ, E. \& GARCíA, R. (2000). Análisis de la interacción maestro-alumnos durante la resolución de problemas aritméticos. Cultura E Educación, 17/18, 41-61. 
FusON, K. C. (1992). Research on whole number addition and subtraction. En D. Grouws (Ed.), Handbook of research on mathematics teaching and learning (pp. 243-275). Nueva York: Macmillan.

HatANO, G. \& INAGAKI, K. (1991). Sharing cognition through collective comprehension activity. En L. Resnick, J. Levine \& S. Teasley (Eds.), Perspectives on socially-shared cognition (pp. 331-348). Washington, DC: American Psychological Association.

Hogan, T., Rabinowitz, M. \& Craven, J. A. (2003). Representation in teaching: Inferences from research of expert and novice teachers. Educational Psychologist, 38 (4), 235-247.

KINTSCH, W. (1988). The role of knowledge in discourse comprehension: a Construction-Integration model. Psychological Review, 95 (2), 163-182.

KINTSCH, W. (1998). Comprehension: a paradigm for cognition. Cambridge: Cambridge University Press.

KintsCH, W. \& GreENO, J. (1985). Understanding and solving word arithmetic problems. Psychological Review, 92, 109129.

LEMKE, J. (1997). Aprender a hablar ciencia. Barcelona: Paidós.

LEWIS, A. B. \& MAYER, R. E. (1987). Students's miscomprehension of relational statements in arithmetic word problems. Journal of Educational Psychology, 79 (4), 363-371.

Mercer, N. \& Littleton, K. (2007). Dialogue and the Development of Children's Thinking. A sociocultural approach. Londres: Routledge.

Nathan, M. J., KintSCH, W. \& Young, E. (1992). A theory of algebra-word problem comprehension and its implications for the design of Learning Environments. Cognition and Instruction, 9 (4), 329-389.

NAtHAn, M. J. \& KNUTH, E. J. (2003). A study of whole classroom mathematical discourse and teacher change. Cognition and Instruction, 21 (2), 175-207.

REED, S. K. (1999). Word Problems: Research and curriculum reform. Mahwah, NJ: Lawrence Erlbaum Associates

Reusser, K. (1988). Problem solving beyond the logic of things: Contextual effects on understanding and solving word problems. Instructional Science, 17, 309-338.

RiLEY, M. S. \& GrEENO, J. G. (1988). Developmental analysis of understanding language about quantities of solving problems. Cognition E Instruction, 5 (1), 49-101.

Riley, N. S., Greeno, J. \& HeLler, J. I. (1983). Development of children's problem solving ability in arithmetic. En H. P. Ginsburg (Ed.), The development of mathematical thinking (pp. 153-196). Nueva York: Academic Press.

Rosales, J., ItURRA, C., SÁNCHEZ, E. \& DE SiXTE, R. (2006). El análisis de la práctica educativa. Un análisis de la interacción profesor-alumnos a partir de dos sistemas de análisis diferentes. Infancia y Aprendizaje, 29 (1), 65-90.

Rosales, J., OrRantia, J., Vicente, S., Chamoso, J. M. (2008). Resolution of word problems and interaction in the classroom. A comparison between in-service and pre-service teachers. European Journal of Psychology of Education, 32 (3), $275-294$.

Sánchez, E., García, J. R., Castellano, N., de Sixte, R., Bustos, A. \& García-Rodicio, H. (2008). Qué, cómo y quién: tres dimensiones para analizar la práctica educativa. Cultura E Educación, 20 (1), 95-118

SÁNCHEZ, E. \& RosALES, J. (2005). La práctica educativa. Una revisión a partir del estudio de la interacción profesor-alumnos en el aula. Cultura E Educación, 17 (2), 147-173.

SÁNCHEZ, E., ROSALES, J. \& SUÁREZ, S. (1999). Interacción profesor-alumnos y comprensión de textos. Qué se hace y qué se puede hacer. Cultura E Educación, 14/15, 71-89.

STAUB, F. C. \& REUSSER, K. (1995). The role of presentational structures in understanding and solving mathematical word problems. En C.A. Weaver III, S. Mannes \& C. R. Fletcher (Eds.), Discourse Comprehension: Essays in honour of Walter Kintsch (pp. 285-305). Hillsdale, NJ: Lawrence Erlbaum.

STERN, E. (1993). What makes certain arithmetic word problems involving the comparison of sets so difficult for children? Journal of Educational Psychology, 85, 7-23.

STODOLSKY, S. (1992). La importancia del contenido. Madrid: Paidós.

Verschaffel, L. \& De CORTE, E. (1997). Teaching realistic mathematical modeling in the elementary school: a teaching experiment with fifth graders. Journal for Research in Mathematic Education, 28 (5), 577-601.

Vicente, S., OrRANTIA, J. \& VerSCHAFFEL, L. (2008). Influencia del conocimiento matemático y situacional en la resolución de problemas aritméticos verbales: ayudas textuales y gráficas. Infancia y Aprendizaje, 31 (4), 387-402.

Williams, S. R. \& BAXTER, J. A. (1996). Dilemmas of discourse-oriented teaching in one Middle School Mathematics Classroom. The Elementary School Journal, 97 (1), 21-38. 